\title{
Fixed point theorems on partially ordered topological vector spaces and their applications to equilibrium problems with incomplete preferences
}

Jinlu Li*

Correspondence: jli@shawnee.edu

Department of Mathematics,

Shawnee State University,

Portsmouth, $\mathrm{OH}$ 45662, USA

\section{空 Springer}

\begin{abstract}
In this paper, we examine some properties of chain-complete posets and introduce the concept of universally inductive posets. By applying these properties, we provide several extensions of Abian-Brown fixed point theorem from single-valued mappings to set-valued mappings on chain-complete posets and on compact subsets of partially ordered topological spaces. As applications of these fixed point theorems, we explore the existence of generalized Nash equilibrium for strategic games with partially ordered preferences.
\end{abstract}

MSC: 06F30; 91A06; $91 \mathrm{~A} 18$

Keywords: universally inductive poset; partially ordered topological space; fixed point; generalized Nash equilibrium; strategic game with partially ordered preferences

\section{Introduction}

In game theory, the players (or the decision makers) usually have normal preferences (i.e. completely ordered preferences) on the outcomes of the games. More precisely, in any such game, for any given pair of outcomes, the players can clearly show their preference one to other one, or can identify both outcomes as equally desirable (indifferent between these two outcomes). That is, the utility functions for the players on the outcomes in such games have ranges in totally ordered sets, which can be represented by real valued functions (payoff functions).

In contrast to games with normal preferences, in the real world, there are some games that the utilities for the players on the outcomes are not totally ordered (maybe partially ordered), in which, there are some pairs of distinct outcomes which cannot be identified the preferences between them by the players. We provide an example below to more precisely illustrate this argument (for more details, see [1]).

\subsection{The extended prisoner's dilemma}

Two suspects, designated Suspect 1 and Suspect 2, are held in separate cells without any means of communicating with each other. There are two crimes (I and II) for which these suspects are being held. There is enough evidence to convict each of them of minor offenses related to crimes I and II, but not enough evidence to convict either of them of the

(c) $2014 \mathrm{Li}$; licensee Springer. This is an Open Access article distributed under the terms of the Creative Commons Attribution License (http://creativecommons.org/licenses/by/2.0), which permits unrestricted use, distribution, and reproduction in any medium, provided the original work is properly cited. 
principal crimes I or II unless one of them acts as an informant against the other ('finks') for crime I or II.

If they both stay quiet for both crime I and crime II, then each will be convicted of the minor offenses for both crime I and crime II, and each will spend one year in prison for crime I and will be fined $\$ 10$ for crime II. If only one of them finks for crime I and they both stay quiet for crime II, then the informant will not be charged for crime I but will be fined $\$ 10$ for crime II, and the informant will testify against the other for crime I, who will be convicted of the principal offense for crime I resulting in a three-year prison sentence and be fined $\$ 20$ for crime II. If they both stay quiet for crime I and only one of them finks for crime II, then the informant will not be charged for crime II but will spend one year in prison for crime I, and the informant will testify against the other for crime II, who will be convicted of the principal offense for crime II resulting in a $\$ 30$ fine and be sentenced to two years in prison for crime I. If they both fink for both crimes I and II, then each will spend two years in prison for crime I and be fined $\$ 20$ for crime II.

Every suspect has the following four possible strategies:

QQ, $\quad \mathrm{QF}, \quad \mathrm{FQ}, \quad \mathrm{FF}$,

where the first letter represents the action for crime I and the second letter represents the action for crime II. Then the possible outcomes (payoffs) for this game can be described by the following table, where Suspect 1 plays this game as the row player and Suspect 2 plays this game as the column player. Then this game has the following utilities matrix:

$\begin{array}{ccccc} & \text { QQ } & \text { QF } & \text { FQ } & \text { FF } \\ \text { QQ } & (-1,-10),(-1,-10) & (-2,-30),(-1,0) & (-3,-20),(0,-10) & (-3,-30),(0,0) \\ \text { QF } & (-1,0),(-2,-30) & (-2,-20),(-2,-20) & (-3,-10),(-1,-30) & (-3,-20),(-1,-20) \\ \text { FQ } & (0,-10),(-3,-20) & (-1,-30),(-3,-10) & (-2,-20),(-2,-20) & (-3,-30),(-2,-10) \\ \text { FF } & (0,0),(-3,-30) & (-1,-20),(-3,-20) & (-2,-10),(-3,-30) & (-2,-20),(-2,-20)\end{array}$

In every entry of the above matrix table, the first point is the outcome for Suspect 1 and the second point is for Suspect 2. At each point, the absolute value of the first coordinate equals the number of years that the suspect will spend in prison for crime I and the absolute value of the second coordinate equals the number of dollars the suspect is fined for crime II.

If we assume that, for every suspect, the number of years in prison for crime I and the number of dollars fined for crime II are not substitutable, and then the utilities of the two players are in the 2-dimensional Euclidean space $R^{2}$, which is endowed with the coordinate ordering relation $\succeq^{2}$ on $R^{2}$.

Let $P_{1}, P_{2}$ be the utility functions (payoffs) of Suspect 1 and Suspect 2, respectively. So the preferences of every suspect are not totally ordered. The utility functions $P_{1}$ and $P_{2}$ both take values in $\left(\mathbb{R}^{2} ; \succeq^{2}\right)$. From the above table, we have

$$
P_{1}(\mathrm{QQ}, \mathrm{QQ})=(-1,-10), \quad P_{1}(\mathrm{FQ}, \mathrm{FQ})=(-2,-20), \quad \text { and } \quad P_{1}(\mathrm{QF}, \mathrm{FQ})=(-3,-10) .
$$

It implies

$$
P_{1}(\mathrm{QQ}, \mathrm{QQ}) \succ^{2} P_{1}(\mathrm{FQ}, \mathrm{FQ}) \quad \text { and } \quad P_{1}(\mathrm{FQ}, \mathrm{FQ}) \bowtie^{2} P_{1}(\mathrm{QF}, \mathrm{FQ})
$$


Then we see that Suspect 1 prefers strategic profile (action) (FQ, QQ) to (QQ, QQ), and he prefers strategic profile (QQ, QQ) to (FQ, FQ). But Suspect 1 does not have any preferences between strategic profiles (QF, FQ) and (FQ, FQ), at which, the suspect's utilities are $(-3,-10)$ and $(-2,-20)$, respectively; that is, the utilities for Suspect 1 on strategic profiles $(\mathrm{QF}, \mathrm{FQ})$ and $(\mathrm{FQ}, \mathrm{FQ})$ are not comparable.

In this game, the preferences of the two players are not normal; and therefore, we cannot define the ordinary Nash equilibrium for this game. In [1-5], some strategic games with nonnormal preferences have been studied and the concept of Nash equilibrium with normal preferences has been extended to more general Nash equilibrium with nonnormal preferences.

In the ordinary game theory, fixed point theorems play important roles to prove the existence of Nash equilibrium for strategic games, where the underlying spaces are equipped with topological structures. So in such games, the considered payoff functions are assumed to satisfy some continuity conditions. In contrast to, if the underlying spaces in a game are posets endowed with a partial order, which may not equipped with a topological structure, then the ordinal techniques for proving the existence of Nash equilibrium are not applicable. To overcome this difficulty, in this paper, we develop more fixed point theorems in posets with set-valued mappings, which is applied to solving the Nash equilibrium problems for strategic games with partially ordered preferences.

\section{Partially ordered topological spaces}

In this section, we recall some properties of partially ordered topological spaces. The notations used in this section are derived from Aliprantis and Burkinshaw [6], Carl and Heikkilä [2], Debreu [7], Dunford and Schwartz [8], Li [1], Ok [9], and Ward [10].

Let $(P, \succeq)$ be a poset (i.e. a partially ordered set) and $A$ a nonempty subset of $P$. We say that $A$ is

1. inductive if every chain in $A$ has an upper bound in $A$;

2. chain complete if every chain $C$ in $A$ possess its supremum, denoted by $\vee C$, in $A$.

A real vector space $X$ endowed with a partial order $\succeq$ is called a partially ordered vector space, which is written as $(X, \succeq)$ (it is a poset), if the following (order-linearity) properties hold:

1. $x \succeq y$ implies $x+z \succeq y+z$, for all $x, y, z \in X$.

2. $x \succeq y$ implies $\alpha x \geq \alpha y$, for all $x, y \in X$ and $\alpha \geq 0$.

In this definition, in the underlying vector space, there is only algebraic structure endowed with a partial order $\succeq$, on which there is no topological structure equipped.

Let $(P, \succeq)$ be a poset. For any $z, w \in P$, we denote the following $\succeq$-intervals:

$$
\begin{aligned}
& {[z)=\{x \in P: x \succeq z\}, \quad(w]=\{x \in P: x \preceq w\} \quad \text { and }} \\
& {[z, w]=[z) \cap(w]=\{x \in P: z \preceq x \preceq w\} .}
\end{aligned}
$$

Let $(X, \succeq)$ be a poset equipped with a topology $\tau$ (it is also a topological space). The topology $\tau$ is called a natural topology on $(X, \succeq)$ with respect to the partial order $\succeq$, whenever, for every $z \in P$, the $\succeq$-intervals $[z)$ and $(z]$ are all $\tau$-closed.

A poset $(X, \succeq)$ equipped with a natural topology $\tau$ on $X$ is called a partially ordered topological space; and it is denoted by $(X, \tau, \succeq)$. 
A partially ordered topological vector space is both a partially ordered topological space and a partially ordered vector space.

We say that a Banach space endowed with a partial order is a partially ordered Banach space if it is both a partially ordered vector space and a partially ordered topological space with respect to the norm topology.

Theorem 2.1 Let $(B,\|\cdot\|)$ be a Banach space with the norm $\|\cdot\|$. Let $\succeq$ be a partial order on $B$ satisfying that $(B, \succeq)$ is a partially ordered vector space. Let $\omega$ be the weak topology on $B$ with respect to the norm $\|\cdot\|$. Then the norm topology $\|\cdot\|$ on B is a natural topology with respect to the partial order $\succeq$, if and only if the weak topology $\omega$ on $B$ is a natural topology with respect to $\succeq$.

Proof For every $x \in B$, since $(B, \succeq)$ is a partially ordered vector space, it yields that the $\succeq$ intervals $[x)$ and $(x]$ are convex subsets of $B$. Then this theorem immediately follows from the fact that every convex subset of a Banach space is closed in the norm topology if and only if it is closed in the weak topology (see Theorem 1.2.12 in Takahashi [11]).

Lemma 2.2 (Ok [9]) Let $(X, d, \succeq)$ be a partially ordered compact metric space. Then $(X, \succeq)$ is a chain-complete poset.

Next, we extend Lemma 2.2 from partially ordered compact metric space to compact subsets of partially ordered topological spaces.

Theorem 2.3 Every nonempty compact subset of a partially ordered Hausdorff topological space is chain complete.

Proof Let $(X, \tau, \succeq)$ be a partially ordered Hausdorff topological space with natural topology $\tau$. Let $D$ be a nonempty compact subset of $X$. Pick an arbitrary chain $C$ in $D$. Let $\operatorname{cl}(C)$ denote the $\tau$-closure of $C$. Since $D$ is compact, so $\operatorname{cl}(C) \subseteq D$; and $\operatorname{cl}(C)$ is also a compact subset of $X$. At first, we show that $\operatorname{cl}(C)$ is also a chain in $D$. To this end, take any arbitrary pair of elements $x, y \in \operatorname{cl}(C)$. Then there are sequences $\left\{x_{n}\right\}$ and $\left\{y_{n}\right\}$ in $C$ such that $x_{n} \rightarrow x$ and $y_{n} \rightarrow y$, as $n \rightarrow \infty$. Since $C$ is a totally ordered subset of $P$, then $x_{n} \preceq y_{n}$ or $y_{n} \preceq x_{n}$ holds for every $n=1,2,3, \ldots$. Hence there must be subsequences of $\left\{x_{n}\right\}$ and $\left\{y_{n}\right\}$, without loss of generality, assuming itself, such that $x_{n} \leq y_{n}$ holds for every $n=1,2,3, \ldots$.

We claim that any sequence $\left\{z_{n}\right\}$ in a totally ordered set $C$ contains a totally ordered subsequence. In fact, we define two sets of positive integers below:

$L=\left\{n:\right.$ there are infinitely many $m$ such that $\left.z_{m} \preceq z_{n}\right\}$

and

$M=\left\{n:\right.$ there are infinitely many $m$ such that $\left.z_{m} \succeq z_{n}\right\}$.

It is clear that at least one of the $L$ and $M$ must be infinite. If $L$ is infinite, then we can select an order decreasing subsequence (it is totally ordered) of $\left\{x_{n}\right\}$; and if $M$ is infinite, then we can select an order increasing subsequence (it is also totally ordered) of $\left\{x_{n}\right\}$. 
So, since $\left\{x_{n}\right\}$ and $\left\{y_{n}\right\}$ are sequences in the chain $C$, we can select order monotonic subsequences from $\left\{x_{n}\right\}$ and $\left\{y_{n}\right\}$, respectively. Without loss of generality, we assume that $\left\{x_{n}\right\}$ and $\left\{y_{n}\right\}$ are both sub-chains of the chain $C$; that is, $x_{n} \uparrow$ (order increasing) and $y_{n} \uparrow$ (similarly, we can prove the result for the cases $x_{n} \uparrow$ and $y_{n} \downarrow$ (order decreasing), or, $x_{n} \downarrow$ and $\left.y_{n} \downarrow\right)$.

Then from the assumption that $x_{n} \preceq y_{n}$ holds for every $n=1,2,3, \ldots$, for every fixed positive integer $n$, we have $y_{m} \succeq x_{n}$, if $m \geq n$. For the given fixed positive integer $n$, the following $\succeq$-interval:

$$
\left[x_{n}\right) \cap D=\left\{x \in D: x \succeq x_{n}\right\}=\left\{x \in X: x \succeq x_{n}\right\} \cap D
$$

is $\tau$-closed (we assumed that the topology $\tau$ is a natural topology with respect to the endowed partial order $\succeq)$. From $y_{m} \rightarrow y$, as $m \rightarrow \infty$ and $y_{m} \in\left[x_{n}\right)_{D}$, for $m \geq n$, it implies $y \in\left[x_{n}\right)_{D}$; that is

$$
y \succeq x_{n}, \quad \text { for every fixed positive integer } n=1,2, \ldots
$$

That is, $x_{n} \in(y] \cap D$, for $n=1,2, \ldots$. Since $(y] \cap D$ is $\tau$-closed, from $x_{n} \rightarrow x$, as $n \rightarrow \infty$, it yields that $x \in(y] \cap D$; that is, $y \succeq x$. Hence $\operatorname{cl}(C)$ is also a chain in $D$.

Take any arbitrary chain $\left\{x_{\alpha}\right\}$ in $\operatorname{cl}(C)$. Let $\operatorname{cl}\left(\left\{x_{\alpha}\right\}\right)$ denote the $\tau$-closure of $\left\{x_{\alpha}\right\}$. From the above argument, we see that $\operatorname{cl}\left(\left\{x_{\alpha}\right\}\right)$ is also a chain. It is clear that $\operatorname{cl}\left(\left\{x_{\alpha}\right\}\right) \subseteq \operatorname{cl}(C)$. We show that $\left\{x_{\alpha}\right\}$ has an upper bound in $\operatorname{cl}(C)$. To this end, it is sufficient to show that $\operatorname{cl}\left(\left\{x_{\alpha}\right\}\right)$ has a maximum element in $\operatorname{cl}\left(\left\{x_{\alpha}\right\}\right) \subseteq \operatorname{cl}(C)$. For every $x_{\beta} \in \operatorname{cl}\left(\left\{x_{\alpha}\right\}\right)$, let

$$
D_{\beta}=\left\{z \in X: z \prec x_{\beta} \text { or } z \bowtie x_{\beta}\right\} .
$$

Notice that $D_{\beta}$ is the complementary set of the order interval $\left[x_{\beta}\right)$ that is a $\tau$-closed subset of $D$; hence, $D_{\beta}$ is $\tau$-open in $X$. Rest of the proof for $\left\{x_{\alpha}\right\}$ has an upper bound in $\operatorname{cl}(C)$ is divided into two cases below:

Case 1. The collection $\left\{D_{\beta}: x_{\beta} \in \operatorname{cl}\left(\left\{x_{\alpha}\right\}\right)\right\}$ does not form an open covering of the set $\operatorname{cl}\left(\left\{x_{\alpha}\right\}\right)$, that is,

$$
\operatorname{cl}\left(\left\{x_{\alpha}\right\}\right) \nsubseteq \bigcup\left\{D_{\beta}: x_{\beta} \in \operatorname{cl}\left(\left\{x_{\alpha}\right\}\right)\right\} .
$$

It implies that there is $x_{0} \in \operatorname{cl}\left(\left\{x_{\alpha}\right\}\right)$ such that

$$
x_{0} \notin D_{\beta}=\left\{z \in X: z \prec x_{\beta} \text { or } z \bowtie x_{\beta}\right\}, \quad \text { for every } x_{\beta} \in \operatorname{cl}\left(\left\{x_{\alpha}\right\}\right) .
$$

From (2), it yields

$x_{0} \succeq x_{\beta}, \quad$ for every $x_{\beta} \in \operatorname{cl}\left(\left\{x_{\alpha}\right\}\right)$.

Hence, $x_{0}$, which belongs to $\operatorname{cl}\left(\left\{x_{\alpha}\right\}\right)$, is a maximum element of $\operatorname{cl}\left(\left\{x_{\alpha}\right\}\right)$.

Case 2. The collection $\left\{D_{\beta}: x_{\beta} \in \operatorname{cl}\left(\left\{x_{\alpha}\right\}\right)\right\}$ forms an open covering of the $\operatorname{set} \operatorname{cl}\left(\left\{x_{\alpha}\right\}\right)$, that is,

$$
\operatorname{cl}\left(\left\{x_{\alpha}\right\}\right) \subseteq \bigcup\left\{D_{\beta}: x_{\beta} \in \operatorname{cl}\left(\left\{x_{\alpha}\right\}\right)\right\}
$$


Since $\operatorname{cl}\left(\left\{x_{\alpha}\right\}\right)$ is a $\tau$-closed subset of the compact subset $D$ of the Hausdorff topological space $(X, \tau)$, then it is a $\tau$-compact subspace of $D$. Hence there is a finite subset $\left\{D_{j}: j=\right.$ $1,2, \ldots, m\}$ of $\left\{D_{\beta}: x_{\beta} \in \operatorname{cl}\left(\left\{x_{\alpha}\right\}\right)\right\}$ that covers $\operatorname{cl}\left(\left\{x_{\alpha}\right\}\right)$, for some positive integer $m$, where $D_{j}$ is defined by (1) with an element $x_{j} \in \operatorname{cl}\left(\left\{x_{\alpha}\right\}\right)$. That is,

$$
\operatorname{cl}\left(\left\{x_{\alpha}\right\}\right) \subseteq \bigcup\left\{D_{j}: j=1,2,3, \ldots, m\right\} .
$$

Since $\operatorname{cl}\left(\left\{x_{\alpha}\right\}\right)$ is totally ordered, and $\left\{x_{j}: j=1,2,3, \ldots, m\right\} \subseteq \operatorname{cl}\left(\left\{x_{\alpha}\right\}\right)$, there is a maximum element of the finite subset $\left\{x_{j}: j=1,2,3, \ldots, m\right\}$. Without loss of generality, assuming that $x_{m}$ is the maximum element of $\left\{x_{j}: j=1,2,3, \ldots, m\right\}$, that is,

$$
x_{j} \preceq x_{m}, \quad \text { for } j=1,2,3, \ldots, m \text {. }
$$

For any given $x \in \operatorname{cl}\left(\left\{x_{\alpha}\right\}\right)$, from (3), there is $j=1,2,3, \ldots, m$, such that $x \in D_{j}$; that is, $x \prec x_{j}$ or $x \bowtie x_{j}$. Since both $x$ and $x_{j}$ are in $\operatorname{cl}\left(\left\{x_{\alpha}\right\}\right)$ that is totally ordered, from (4), we must have

$$
x \prec x_{j} \preceq x_{m}, \quad \text { for some } j=1,2,3, \ldots, m \text {. }
$$

Hence, this element $x_{m} \in \operatorname{cl}\left(\left\{x_{\alpha}\right\}\right)$ is the maximum element of $\operatorname{cl}\left(\left\{x_{\alpha}\right\}\right)$ and $x_{m} \in \operatorname{cl}\left(\left\{x_{\alpha}\right\}\right) \subseteq$ $\operatorname{cl}(C)$.

Combing the above two cases, we find that every chain $\left\{x_{\alpha}\right\}$ in $\mathrm{cl}(C)$ has an upper bound in $\operatorname{cl}(C)$. Hence $\operatorname{cl}(C)$ is inductive.

From Zorn's lemma, $\mathrm{cl}(C)$ has a maximal element. Since $\mathrm{cl}(C)$ is totally ordered, its maximal element must be unique that is its maximum element. We denote it by $x^{*}$. Since $C \subseteq \operatorname{cl}(C)$, it implies that $x^{*}$ is an upper bound of the chain $C$ in $D$. Next we claim

$$
x^{*}=\vee C .
$$

To prove (5), suppose that $y \in D$ is also an upper bound of $C$, that is, $y \geq x$, for every $x \in C$. From $x^{*} \in \operatorname{cl}(C)$, there exists a sequence $\left\{z_{n}\right\} \subseteq C$ such that

$$
z_{n} \rightarrow x^{*} \quad \text { as } n \rightarrow \infty
$$

Since $y$ is an upper bound of the chain $C$ in $D$, and $\left\{z_{n}\right\} \subseteq C$, it yields $\left\{z_{n}\right\} \subseteq(y]$. From the assumption that the topology $\tau$ is a natural topology with respect to the endowed partial order $\succeq,(y]$ is $\tau$-closed. Then (6) implies $x^{*} \in(y]$, that is, $x^{*} \preceq y$, which shows (5). It completes the proof of this theorem.

Following the above theorems, we give some examples of partially ordered topological vector spaces and chain complete subsets. At first, we consider some special partial orders on vector spaces, which are induced by some closed cones in these spaces, which is especially important in the applications to the vector variational analysis.

Let $K$ be a nonempty subset of a vector space $X . K$ is called a cone in $X$ if it satisfies $K \neq\{0\}, a K \subseteq K$, for any nonnegative number $a$ and $(-K) \cap K=\{0\}$. An ordering relation $\succeq_{K}$ on $X$ induced by a cone $K$ in $X$ is define thus: for any $x, y \in X$,

$$
x \succeq_{K} y \quad \text { if and only if } \quad x-y \in K \text {. }
$$


It follows that, for every $x \in X$, the $\succeq_{K}$-intervals are given by

$$
[x)=x+K \quad \text { and } \quad(x]=x-K .
$$

The following results are useful and well known, which can be easily proved by applying (7). We list it as a proposition.

Proposition 2.4 Let $X$ be a vector space and let $K$ be a cone in $X$. We have

1. If $K$ is convex, then the ordering relation $\succeq_{K}$ on $X$ induced by $K$ is a partial order and $\left(X, \succeq_{K}\right)$ is a partially ordered vector space;

2. If $(X, \tau)$ is a topological vector space and $K$ is closed and convex, then $\left(X, \tau, \succeq_{K}\right)$ is a partially ordered topological vector space;

3. If $(X,\|\cdot\|)$ is a Banach space and $K$ is closed and convex, then $\left(X,\|\cdot\|, \succeq_{K}\right)$ is a partially ordered Banach space with the norm topology; and $\left(X, \omega, \succeq_{K}\right)$ is also a partially ordered topological vector space with the weak topology $\omega$.

Theorem 2.5 Let $(X, \tau, \succeq)$ be a partially ordered topological space. Let $\left\{x_{n}\right\}$ be an $\succeq$ increasing ( $\succeq$-decreasing) sequence (sequential chain) in $X$.If $\vee\left\{x_{n}\right\}\left(\wedge\left\{x_{n}\right\}\right)$ exists and $\left\{x_{n}\right\}$ is convergent, then $x_{n} \rightarrow \vee\left\{x_{n}\right\}\left(\wedge\left\{x_{n}\right\}\right)$, as $n \rightarrow \infty$.

Proof For the case that $\left\{x_{n}\right\}$ is an $\succeq$-increasing sequence, suppose that $\vee\left\{x_{n}\right\}$ exists and that $x_{n} \rightarrow x$, as $n \rightarrow \infty$, for some $x \in X$. Since $\tau$ is a natural topology with respect to the partial order $\succeq$, then the order interval $\left(\vee\left\{x_{n}\right\}\right]$ is $\tau$-closed. Hence we must have $x \in$ $\left(\vee\left\{x_{n}\right\}\right]$. It implies

$$
x \preceq \vee\left\{x_{n}\right\} .
$$

We claim that $x_{n} \preceq x$, for all $n$. In fact, for every fixed $m$, from the fact that $\tau$ is a natural topology with respect to the partial order $\succeq$ again, the order interval $\left[x_{m}\right)$ is $\tau$-closed. Since $\left\{x_{n}\right\}$ is an $\succeq$-increasing sequence, then $\left\{x_{n}: n \geq m\right\} \subseteq\left[x_{m}\right)$. From the assumption that $x_{n} \rightarrow x$, as $n \rightarrow \infty$, it implies $x \in\left[x_{m}\right)$; that is, $x \succeq x_{m}$, for all $m$. Hence $x$ is an upper bound of $\left\{x_{n}\right\}$. By (8), it implies $x=\vee\left\{x_{n}\right\}$. We obtain $x_{n} \rightarrow \vee\left\{x_{n}\right\}$, as $n \rightarrow \infty$. We can similarly show this theorem for the case that $\left\{x_{n}\right\}$ is an $\succeq$-decreasing sequence.

The following corollaries immediately follow from Theorem 2.3.

Corollary 2.6 Let $(X, \succeq)$ be a partially ordered reflexive Banach space. Then, for any nonempty bounded norm closed and convex subset $D$ of $X,(D, \succeq)$ is a chain complete poset.

Corollary 2.7 Let $X$ be a reflexive Banach space and let $K$ be a closed convex cone of $X$. Let $\succeq_{K}$ be the partial order on $X$ induced by the closed cone $K$. Then, for any nonempty bounded norm closed and convex subset $D$ of $X,\left(D, \succeq_{K}\right)$ is a chain-complete poset.

\section{Several fixed point theorems on partially ordered topological spaces}

Let $\left(X, \succeq^{X}\right)$ and $\left(U, \succeq^{U}\right)$ be posets and let $F: X \rightarrow 2^{U} \backslash\{\varnothing\}$ be a set-valued mapping. $F$ is said to be isotone or order-increasing upward whenever $x \preceq^{X} y$ in $X$ implies that, for any $z \in F(x)$, there is a $w \in F(y)$ such that $z \preceq^{U} w$. F is said to be order-increasing downward 
whenever if $x \preceq^{X} y$ in $X$ implies that, for any $w \in F(y)$, there is a $z \in F(x)$ such that $z \preceq^{U} w$. If $F$ is both of order-increasing upward and order-increasing downward, then $F$ is said to be order-increasing.

The order-increasing property of mappings plays important roles in the fixed point theory on posets. In [1], Li provided several fixed point theorems that are extensions of Abian-Brown fixed point theorem from single-valued mappings to set-valued mappings on posets, which is equipped with neither a topology structure, nor an algebraic structure. In this section, we introduce the concept of universally inductive posets and improve the results provided in [1].

Definition 3.1 A nonempty subset $A$ of a poset $(P, \succeq)$ is said to be universally inductive in $P$ whenever, any given chain $\left\{x_{\alpha}\right\} \subseteq P$ satisfying that every element $x_{\beta} \in\left\{x_{\alpha}\right\}$ has an upper cover in $A$ has an upper bound in $A$.

Lemma 3.2 Every inductive subset $A$ in a chain complete poset such that $A$ has finite number of maximal elements is universally inductive.

Proof See the proof of Theorem 3.2 in [1].

It is clear that every universally inductive poset is an inductive poset. Next we provide a counter example of poset that is inductive, but not universally inductive.

Example 3.3 Let $\left(R^{2}, \succeq^{2}\right)$ denote the partially ordered topological space with the coordinative partial order and the ordinal topology on the 2-Euclidean space $R^{2}$. Let $A=$ $\{(x, 3-x): 0 \leq x<1\}$. Then $A$ is inductive, but not universally inductive.

Proof Clearly, $A$ is not compact. Notice that every element of $A$ is a maximal element in $A$; and therefore, $A$ is inductive. Take a chain $C=\{(x, x): 0 \leq x<1\}$ in $R^{2}$. It is clear that, for every $(x, x) \in C$, there is $(x, 3-x) \in A$ such that $(x, x) \preceq^{2}(x, 3-x)$. But $C$ does not have an upper bound in $A$. Hence, $A$ is not universally inductive.

Theorem 3.4 Let $(P, \succeq)$ be a chain-complete poset and let $F: P \rightarrow 2^{P} \backslash\{\varnothing\}$ be a set-valued mapping. Assume F satisfies the following three conditions:

A1 $F$ is order-increasing upward;

$\mathrm{A}^{\prime}(F(x), \succeq)$ is universally inductive in $P$, for every $x \in P$;

A3 There is an element $y$ in $P$ with $y \preceq v$, for some $v \in F(y)$.

Then F has a fixed point.

Proof Define a subset $A$ of $P$ as follows:

$A=\{x \in P:$ there is $z \in F(x)$ such that $x \preceq z\}$.

From condition 3, $y \in A$; and therefore, $A$ is a nonempty subset of $P$. At first, we show that, for any $x \in A$, if $z \in F(x)$ such that $x \preceq z$, then $z \in A$. To this end, from $x \preceq z$ and $z \in F(x)$, applying condition A1, it implies that there is $u \in F(z)$ such that $z \preceq u$. It follows that $z \in A$. 
Next, we show that $A$ is inductive. For an arbitrary chain $\left\{x_{\alpha}\right\} \subseteq A$, since $P$ is chain complete, it follows that $\vee\left\{x_{\alpha}\right\}$ exists. For every $x_{\beta} \in\left\{x_{\alpha}\right\} \subseteq A$, there is $u_{\beta} \in F\left(x_{\beta}\right)$ with $x_{\beta} \preceq u_{\beta}$. From $x_{\beta} \preceq \vee\left\{x_{\alpha}\right\}$ and condition A1, there is $w_{\beta} \in F\left(\vee\left\{x_{\alpha}\right\}\right)$ such that

$$
x_{\beta} \preceq u_{\beta} \preceq w_{\beta}, \quad \text { for every } x_{\beta} \in\left\{x_{\alpha}\right\}
$$

By condition $\mathrm{A} 2^{\prime}, F\left(\vee\left\{x_{\alpha}\right\}\right)$ is universally inductive. From (9), it implies that $\left\{x_{\alpha}\right\}$ has an upper bound in $F\left(\vee\left\{x_{\alpha}\right\}\right)$, say $w \in F\left(\vee\left\{x_{\alpha}\right\}\right)$. That is

$$
x_{\beta} \preceq w, \quad \text { for every } x_{\beta} \in\left\{x_{\alpha}\right\} .
$$

It yields $\vee\left\{x_{\alpha}\right\} \preceq w \in F\left(\vee\left\{x_{\alpha}\right\}\right)$. It immediately follows that $\vee\left\{x_{\alpha}\right\} \in A$. Hence $A$ is chain complete; and therefore, $A$ is an inductive subset of $P$.

Applying Zorn's lemma, $A$ has a maximal element $x^{*}$. The definition of $A$ implies that there is $z \in F\left(x^{*}\right)$ such that $x^{*} \preceq z$. From the above proof, we obtain $z \in A$. Since $x^{*}$ is a maximal element of $A$. Then we must have $x^{*}=z$. Hence, $x^{*}$ is a fixed point of $F$.

As consequences of Theorem 3.4 and Lemma 3.2, we have the following results.

Theorem 3.5 (Theorems $3.1[1])$ Let $(P, \succeq)$ be a chain-complete poset and let $F: P \rightarrow$ $2^{P} \backslash\{\varnothing\}$ be a set-valued mapping satisfying the following three conditions:

A1 $F$ is order-increasing upward;

A2 $(F(x), \succeq)$ is inductive with finite number of maximal elements, for every $x \in P$;

A3 there is an element $y$ in $P$ with $y \preceq v$, for some $v \in F(y)$.

Then F has a fixed point.

In Theorems 3.4 and 3.5, the underlying spaces are posets, which is just endowed with a partial order; and it is equipped with neither a topological structure, nor an algebraic structure. Therefore neither continuity, nor linearity for the considered mappings in these theorems is applied.

Next, we provide some examples of universally inductive sets and investigate some more practical conditions for set-valued mappings to have fixed point in partially ordered topological spaces, where the continuity condition is not applied to the considered mappings.

Lemma 3.6 Every nonempty compact subset of a partially ordered Hausdorff topological space is universally inductive.

Proof Let $(X, \tau, \succeq)$ be a partially ordered Hausdorff topological space with natural topology $\tau$. Let $A$ be a nonempty compact subset of $(X, \tau, \succeq)$. From Theorem $2.3,(A, \succeq)$ is a chain complete subset of $(X, \tau, \succeq)$. Take an arbitrary chain $\left\{x_{\alpha}\right\} \subseteq P$ satisfying that, for every element $x_{\beta} \in\left\{x_{\alpha}\right\}$, there is an element $y_{\beta} \in A$ with $x_{\beta} \preceq y_{\beta} \in A$. Then from the fact that $\tau$ is a natural topology with respect to the partial order $\succeq$ on $X$, it implies that the set $\left[x_{\beta}\right) \cap A$ is a nonempty closed subset of $A$. From the totally ordered property of $\left\{x_{\alpha}\right\}$, it follows that the net $\left\{\left(x_{\alpha}\right) \cap A\right\}$ is also a totally ordered net in $2^{A}$ with respect to the inclusion order. Hence the intersection of any finite number of elements in $\left\{\left[x_{\alpha}\right) \cap A\right\}$ is nonempty. 
So the family of closed subsets $\left\{\left[x_{\alpha}\right) \cap A\right\}$ satisfies the finite intersection condition on the compact set $A$. Then we have

$$
\bigcap_{\alpha}\left(\left[x_{\alpha}\right)\right) \cap A=\bigcap_{\alpha}\left(\left[x_{\alpha}\right) \cap A\right) \neq \varnothing .
$$

Every element in $\bigcap_{\alpha}\left(\left[x_{\alpha}\right)\right) \cap A$ is an upper bound of the chain $\left\{x_{\alpha}\right\}$ in $A$. It proves this lemma.

By applying Proposition 2.4, as consequences of Theorem 3.4 and Lemma 3.6 to ordered topological space, we obtain the following results.

Corollary 3.7 Let $(X, \tau, \succeq)$ be a partially ordered topological space and let $C$ be a nonempty compact subset of $X$. Let $F: C \rightarrow 2^{C} \backslash\{\varnothing\}$ be a set-valued mapping. Assume $F$ satisfies the conditions A1, A3 in Theorem 3.4 and the following condition:

A2" $F(x)$ is closed, for every $x \in P$.

Then F has a fixed point.

Proof From Theorem 2.3, the poset $(C, \succeq)$ is chain-complete. By condition A2", $F(x)$ is a closed subset of a compact set $C$, and then it is compact. From Lemma 3.6, $F(x)$ is universally inductive, for every $x \in P$. Then this corollary follows from Theorem 3.4 immediately.

Corollary 3.8 Let $(X,\|\cdot\|, \succeq)$ be a partially ordered reflexive Banach space and let $C$ be a nonempty bounded closed and convex subset of X. Let $F: C \rightarrow 2^{C} \backslash\{\varnothing\}$ be a set-valued mapping. Assume $F$ satisfies conditions A1, A3 in Theorem 3.4 and the following condition:

A2'" $F(x)$ is closed and convex, for every $x \in P$.

Then F has a fixed point.

Proof Since $C$ is a nonempty bounded closed and convex subset of the reflexive Banach space $X$, it is a weakly compact subset of $(X, \omega, \succeq)$. From Proposition 2.4 , it is also a partially ordered vector space with respect to the weak topology $\omega$ on $X$. By Theorem 2.3, $(C, \succeq)$ is a chain complete poset. The condition $\mathrm{A} 2^{\prime \prime \prime}$ in this corollary implies that $F(x)$ is a weakly compact subset of the bounded closed and convex subset $C$, for every $x \in P$. Then this corollary immediately follows from Lemma 3.6 and Theorem 3.4.

To finish this section, we make some remarks on the results provided in this section.

Remark 3.9 In contrast to the definition of universally inductive poset in Definition 3.1, we can consider reversed universally inductive posets, order-decreasing upward (downward) set-valued mappings. Then we can explore some fixed point theorems similar to Theorem 3.4 with respect to order-decreasing upward (downward) set-valued mappings with values of reversed universally inductive posets. 


\section{Generalized Nash equilibrium of strategic game with partially ordered preferences}

In this section, we study some strategic and noncooperative games, in which the utilities of the players are not totally ordered; that is, the players do not have the normal preferences on the outcomes of this game, which is called a nonmonetized strategic game in this paper. In $[1,3]$ and $[4,5]$, such games are called nonmonetized noncooperative games. The word 'nonmonetized' refers that the preferences of the players are not normal and cannot be represented by real functions.

Definition 4.1 Let $n$ be a positive integer greater than 1. An $n$-person nonmonetized strategic game consists of the following elements:

1. a set of $n$ players, which is denoted by $N=\{1,2, \ldots, n\}$;

2. for every player $i=1,2, \ldots, n$, his set of strategies $\left(S_{i}, \succeq_{i}\right)$ is a poset. The collection of profiles of strategies is denoted by $S=S_{1} \times S_{2} \times \cdots \times S_{n}$;

3. an outcome space $\left(U ; \succeq^{U}\right)$ that is a poset;

4. for every player $i=1,2, \ldots, n$, his utility function (payoff) $P_{i}$ is a mapping from $S_{1} \times S_{2} \times \cdots \times S_{n}$ to $\left(U ; \succeq^{U}\right)$. We write $P=\left\{P_{1}, P_{2}, \ldots, P_{n}\right\}$.

This game is denoted by $\Gamma=(N, S, P, U)$.

A $n$-person nonmonetized strategic game $\Gamma=(N, S, P, U)$ is a noncooperative game. The rule for playing a game $\Gamma=(N, S, P, U)$ is that when all $n$ players simultaneously and independently choose their own strategies $x_{1}, x_{2}, \ldots, x_{n}$, respectively, to act, where $x_{i} \in S_{i}$, for $i=1,2, \ldots, n$, player $i$ will receive his utility (payoff) $P_{i}\left(x_{1}, x_{2}, \ldots, x_{n}\right) \in U$.

For any $x=\left(x_{1}, x_{2}, \ldots, x_{n}\right) \in S$, and for every $i=1,2, \ldots, n$, as usual, we denote

$$
x_{-i}:=\left(x_{1}, x_{2}, \ldots, x_{i-1}, x_{i+1}, \ldots, x_{n}\right) \quad \text { and } \quad S_{-i}:=S_{1} \times S_{2} \times \cdots \times S_{i-1} \times S_{i+1} \times \cdots \times S_{n} .
$$

Then $x_{-i} \in S_{-i}$ and $x$ can be simply written as $x=\left(x_{i}, x_{-i}\right)$. Moreover, we denote

$$
P_{i}\left(S_{i}, x_{-i}\right):=\left\{P_{i}\left(t_{i}, x_{-i}\right): t_{i} \in S_{i}\right\}, \quad \text { for all } x_{-i} \in S_{-i} .
$$

For the convenience, we write $S_{-0}:=S=S_{1} \times S_{2} \times \cdots \times S_{n}$.

Now we extend the concept of Nash equilibrium of strategic games to generalized and extended Nash equilibrium of nonmonetized strategic games.

Definition 4.2 In an $n$-person nonmonetized strategic game $\Gamma=(N, S, P, U)$, a profile of strategies $\left(\tilde{x}_{1}, \tilde{x}_{2}, \ldots, \tilde{x}_{n}\right) \in S_{1} \times S_{2} \times \cdots \times S_{n}$ is called

1. a generalized Nash equilibrium of this game if and only if

$$
P_{i}\left(x_{i}, \tilde{x}_{-i}\right) \preceq^{U} P_{i}\left(\tilde{x}_{i}, \tilde{x}_{-i}\right), \quad \text { for all } x_{i} \in S_{i}, \text { for every } i=1,2, \ldots, n \text {; }
$$

2. an extended Nash equilibrium of this game if and only if

$$
P_{i}\left(x_{i}, \tilde{x}_{-i}\right) \nsucc^{U} P_{i}\left(\tilde{x}_{i}, \tilde{x}_{-i}\right), \quad \text { for all } x_{i} \in S_{i} \text {, for every } i=1,2, \ldots, n \text {. }
$$

It is clear that any generalized Nash equilibrium of a $n$-person nonmonetized strategic game is an extended Nash equilibrium of this game; and the converse may not be true. 
Lemma 4.3 Let $\Gamma=(N, S, P, U)$ be a nonmonetized strategic game. For any fixed $i=$ $0,1,2, \ldots, n$, we define a binary relation $\succeq^{-i}$ on $S_{-i} \times S_{-i}$ as below: for any $x_{-i}, y_{-i} \in S_{-i}$ with $x_{-i}=\left(x_{1}, x_{2}, \ldots, x_{i-1}, x_{i+1}, \ldots, x_{n}\right)$ and $y_{-i}=\left(y_{1}, y_{2}, \ldots, y_{i-1}, y_{i+1}, \ldots, y_{n}\right)$

$$
x_{-i} \succeq^{-i} y_{-i} \quad \text { if and only if } \quad x_{j} \succeq_{j} y_{j}, \quad \text { for all } j=1,2, \ldots, i-1, i+1, \ldots, n \text {. }
$$

Then $\left(S_{-i}, \succeq^{-i}\right)$ is a poset. Furthermore, if for every $j=1,2, \ldots, i-1, i+1, \ldots, n,\left(S_{j}, \succeq_{j}\right)$ is a chain complete (an inductive or a universally inductive) poset, then $\left(S_{-i}, \succeq^{-i}\right)$ is also a chain complete (an inductive or a universally inductive) poset.

Proof The proof is straightforward and is omitted here.

For convenience, $\left(S_{-0}, \succeq^{-0}\right)$ is written as $\left(S, \succeq^{S}\right)$.

Definition 4.4 In a $n$-person nonmonetized strategic game $\Gamma=(N, S, P, U)$, we define

1. a set-valued mapping $B_{i}: S_{-i} \rightarrow 2^{S_{i}}$

$$
B_{i}\left(x_{-i}\right)=\left\{z_{i} \in S_{i}: P_{i}\left(z_{i}, x_{-i}\right) \text { is a maximal element of } P_{i}\left(S_{i}, x_{-i}\right)\right\}, \quad \text { for all } x_{-i} \in S_{-i} .
$$

$B_{i}$ is called the maximal response function for player $i$, for $i=1,2, \ldots, n$;

2. a set-valued mapping $\beta_{i}: S_{-i} \rightarrow 2^{S_{i}}$

$$
\beta_{i}\left(x_{-i}\right)=\left\{z_{i} \in S_{i}: P_{i}\left(z_{i}, x_{-i}\right) \text { is the greatest element of } P_{i}\left(S_{i}, x_{-i}\right)\right\}, \quad \text { for all } x_{-i} \in S_{-i} .
$$

$\beta_{i}$ is called the best response function for player $i$, for $i=1,2, \ldots, n$.

In case if $B_{i}\left(x_{-i}\right) \neq \varnothing$, for all $x_{-i} \in S_{-i}$, then, as a set-valued mapping, $B_{i}$ is order-increasing upward on $S_{-i}$, if, for any $x_{-i}, y_{-i} \in S_{-i}$ with $x_{-i} \preceq^{-i} y_{-i}$ and for any $z_{i} \in B_{i}\left(x_{-i}\right)$, there is $w_{i} \in B_{i}\left(y_{-i}\right)$ such that $z_{i} \preceq_{i} w_{i}$.

Theorem 4.5 Let $\Gamma=(N, S, P, U)$ be an n-person nonmonetized strategic game. Suppose that $\left(S_{i}, \succeq_{i}\right)$ is a chain complete poset, for $i=1,2, \ldots, n$. Assume, for every player $i$, the following conditions hold:

1. $P_{i}\left(S_{i}, x_{-i}\right)$ is an inductive subset of $\left(U, \succeq^{U}\right)$, for every $x_{-i} \in S_{-i}$;

2. the maximal response function $B_{i}: S_{-i} \rightarrow 2^{S_{i}} \backslash\{\varnothing\}$ is order-increasing upward on $\left(S_{-i}, \succeq^{-i}\right)$ with universally inductive values;

3. there are elements $a=\left(a_{1}, a_{2}, \ldots, a_{n}\right)$ and $b=\left(b_{1}, b_{2}, \ldots, b_{n}\right)$ in $S$ with $a \preceq^{S} b$ satisfying

$$
a_{i} \in B_{i}\left(b_{-i}\right), \quad \text { for } i=1,2, \ldots, n \text {. }
$$

Then this game $\Gamma$ has an extended Nash equilibrium.

Proof From assumption 1, and applying Zorn's lemma, $P_{i}\left(S_{i}, x_{-i}\right)$ has at least one maximal element in $P_{i}\left(S_{i}, x_{-i}\right)$. It implies that $B_{i}\left(x_{-i}\right) \neq \varnothing$, for all $x_{-i} \in S_{-i}$.

Notice $x=\left(x_{i}, x_{-i}\right)$, for every $x=\left(x_{1}, x_{2}, \ldots, x_{n}\right) \in S$, and for every $i=1,2, \ldots, n$, and $S$ can be rewritten as $S=\left(S_{i}, S_{-i}\right)$. Then the maximal response function for player $i, B_{i}: S_{-i} \rightarrow$ 
$2^{S_{i}} \backslash\{\varnothing\}$ can be considered as a set-valued mapping from $S$ to $2^{S_{i}} \backslash\{\varnothing\}$ that is defined as

$$
B_{i}(x)=B_{i}\left(x_{-i}\right), \quad \text { for any } x=\left(x_{i}, x_{-i}\right) \in S \text {, for every } i=1,2, \ldots, n \text {. }
$$

Then we define $B: S \rightarrow 2^{S} \backslash\{\varnothing\}$ by $B=B_{1} \times B_{2} \times \cdots \times B_{n}$; that is,

$$
B(x)=\left(B_{1}(x), B_{2}(x), \ldots, B_{n}\left(x_{-n}\right)\right), \quad \text { for any } x \in S .
$$

We show that $B$ is order-increasing upward on $S$. To this end, for any $x, y \in S$ with $x \preceq^{S}$ $y$, and for every $i$, from the definitions of the orders $\preceq^{S}$ on $S$ and $\succeq^{-i}$ on $S_{-i}$, it implies $x_{-i}, y_{-i} \in S_{-i}$ with $x_{-i} \preceq^{-i} y_{-i}$. For any given $z=\left(z_{1}, z_{2}, \ldots, z_{n}\right) \in B(x)$, from (10), we have $z_{i} \in B_{i}\left(x_{-i}\right)$. Since $x_{-i} \preceq^{-i} y_{-i}$, by applying assumption 2 in this theorem, there is $w_{i} \in B_{i}\left(y_{-i}\right)$ with $z_{i} \preceq^{X} w_{i}$, for $i=1,2, \ldots, n$. Let

$$
w=\left(w_{1}, w_{2}, \ldots, w_{n}\right)
$$

It is clearly to be seen that $w \in B(y)$ with $z \preceq^{S} w$. It follows that $B$ is order-increasing upward on $S$.

For every $x=\left(x_{1}, x_{2}, \ldots, x_{n}\right) \in S$, and for every $i=1,2, \ldots, n$, from condition $2, B_{i}\left(x_{-i}\right)$ is a universally inductive subset in $S_{i}$. Then by Lemma 4.3 and from (10), it implies that $B(x)$ is a universally inductive subset in $S$.

From the definition of the mapping $B$, the elements $a, b$ in $S$ given in condition 3 with $a_{i} \in B_{i}\left(b_{-i}\right)$, for $i=1,2, \ldots, n$, satisfy $a \in B(b)$ and $a \preceq^{S} b$. Hence the mapping $B$ satisfies all conditions in Theorem 3.4; and therefore, it has a fixed point, say $v=\left(v_{1}, v_{2}, \ldots, v_{n}\right)$ with $v \in B(v)$. Then, for $i=1,2, \ldots, n, v_{i} \in B_{i}\left(v_{-i}\right)$; that is, $P_{i}\left(v_{i}, v_{-i}\right)$ is a maximal element of $P_{i}\left(S_{i}, v_{-i}\right)$. Hence

$$
P_{i}\left(x_{i}, v_{-i}\right) \nsucc^{U} P_{i}\left(v_{i}, v_{-i}\right), \quad \text { for all } x_{i} \in S_{i} \text { and for } i=1,2, \ldots, n \text {. }
$$

It follows that $v=\left(v_{1}, v_{2}, \ldots, v_{n}\right)$ is an extended Nash equilibrium of this game.

If we replace the maximal response functions $B_{i}$ 's by the best response function $\beta_{i}$ 's in Theorem 4.5, we can get an existence theorem of generalized Nash equilibrium for $n$ person nonmonetized strategic games.

Theorem 4.6 Let $\Gamma=(N, S, P, U)$ be an n-person nonmonetized strategic game. Suppose that $\left(S_{i}, \succeq_{i}\right)$ is a chain complete poset, for $i=1,2, \ldots, n$. Assume, for every player $i$, the following conditions hold:

1. $P_{i}\left(S_{i}, x_{-i}\right)$ is a subset of $\left(U, \succeq^{U}\right)$ and has a greatest element, for every $x_{-i} \in S_{-i}$;

2. The best response function $\beta_{i}: S_{-i} \rightarrow 2^{S_{i}} \backslash\{\varnothing\}$ is order-increasing upward on $\left(S_{-i}, \succeq^{-i}\right)$ with universally inductive values;

3. There are elements $a=\left(a_{1}, a_{2}, \ldots, a_{n}\right)$ and $b=\left(b_{1}, b_{2}, \ldots, b_{n}\right)$ in $S$ with $a \preceq^{S} b$ satisfying

$$
a_{i} \in \beta_{i}\left(b_{-i}\right), \quad \text { for } i=1,2, \ldots, n \text {. }
$$

Then this game $\Gamma$ has a generalized Nash equilibrium. 
In Theorems 4.5 and 4.6, the profiles set and outcomes set are both posets, which is just endowed with a partial order; and it is equipped with neither a topological structure, nor an algebraic structure. Therefore, neither continuity nor linearity for the considered maximal response functions and the best response functions in these theorems is applied. Next we consider some very practical cases that the profiles set and outcomes set are both partially ordered topological spaces, and prove some existence theorems for extended and generalized Nash equilibriums by applying Corollaries 3.7 and 3.8.

Corollary 4.7 Let $\left(X_{i}, \tau_{i}, \succeq_{i}\right)$ be a partially ordered topological space, for $i=1,2, \ldots, n$. Let $\Gamma=(N, S, P, U)$ be an n-person nonmonetized strategic game. Suppose that $S_{i}$ is a compact subset of $X_{i}$, for $i=1,2, \ldots, n$. Assume, for every player $i$, the following conditions hold:

1. $P_{i}\left(S_{i}, x_{-i}\right)$ is an inductive subset of $\left(U, \succeq^{U}\right)$, for every $x_{-i} \in S_{-i}$;

2. the maximal response function $B_{i}: S_{-i} \rightarrow 2^{S_{i}} \backslash\{\varnothing\}$ is order-increasing upward on $\left(S_{-i}, \succeq^{-i}\right)$ with compact values;

3. there are elements $a=\left(a_{1}, a_{2}, \ldots, a_{n}\right)$ and $b=\left(b_{1}, b_{2}, \ldots, b_{n}\right)$ in $S$ with $a \preceq^{S} b$ satisfying

$$
a_{i} \in B_{i}\left(b_{-i}\right), \quad \text { for } i=1,2, \ldots, n \text {. }
$$

Then this game $\Gamma$ has an extended Nash equilibrium.

Corollary 4.8 Let $\left(X_{i}, \tau_{i}, \succeq_{i}\right)$ be a partially ordered topological space, for $i=1,2, \ldots, n$. Let $\Gamma=(N, S, P, U)$ be an n-person nonmonetized strategic game. Suppose that $S_{i}$ is a compact subset of $X_{i}$, for $i=1,2, \ldots, n$. Assume, for every player $i$, the following conditions hold:

1. $P_{i}\left(S_{i}, x_{-i}\right)$ is a subset of $\left(U, \succeq^{U}\right)$ possessing its greatest element, for every $x_{-i} \in S_{-i}$;

2. the best response function $\beta_{i}: S_{-i} \rightarrow 2^{S_{i}} \backslash\{\varnothing\}$ is order-increasing upward on $\left(S_{-i}, \succeq^{-i}\right)$ with compact values;

3. there are elements $a=\left(a_{1}, a_{2}, \ldots, a_{n}\right)$ and $b=\left(b_{1}, b_{2}, \ldots, b_{n}\right)$ in $S$ with $a \preceq^{S} b$ satisfying

$$
a_{i} \in \beta_{i}\left(b_{-i}\right), \quad \text { for } i=1,2, \ldots, n \text {. }
$$

Then this game $\Gamma$ has a generalized Nash equilibrium.

\section{Examples}

Example 5.1 In the example of the extended prisoner's dilemma given in Section 1, it can be easily checked that the action (strategy) profile (FF, FF) is a generalized Nash equilibrium of this game.

In Example 5.1, the set of strategy profiles is finite; and therefore, the ranges of the utility functions are also finite. Next, we spread the extended prisoner's dilemma to a 2-person nonmonetized strategic game with infinitely many strategy profiles and with continuous utility functions.

Example 5.2 (The two arrested venal officials' dilemma) Two suspects, designated Suspect 1 and Suspect 2, are arrested and held in separate cells without any means of communicating with each other. Each of the suspects is potentially guilty of two separate crimes: accepting bribes and/or corruption (abbreviated as $\mathrm{B}$ and $\mathrm{C}$, respectively). In addition, there is enough evidence to convict each suspect of two minor offenses related to B and C, 
respectively: accepting $h$ dollars in bribes and/or embezzling $t$ dollars through corruption, for some $h, t>0$. The prosecutor takes an action against both suspects for bribes accepted up to $H$ and for corruption up to $T$ (in dollars), for some $H>h$ and $T>t$, which are the principal crimes $B$ and $C$. However, there is not enough evidence to convict either suspect of more severely for crimes $B$ and/or $C$ (up to the principal crimes $B$ and/or $C$ ) unless at least one suspect acts as an informant against the other ('finks') for crime B or C.

If both suspects stay quiet, then each will be convicted of the minor offenses for both crime B and C, resulting in fines of $t+2 h$ dollars and jail sentences of $t /(10 \sqrt{T})+h /(\sqrt{H})$ days.

If, for $i=1,2$, simultaneously, Suspect $i$ finks against Suspect $-i$ for crime B by revealing an amount of $x_{i B}$ in addition to $h$ and finks for crime $C$ by revealing an amount of $x_{i C}$ in addition to $t$, then Suspect $i$ will be charged with a reduced penalty if $x_{i B}>x_{-i B}$ and a reduced penalty if $x_{i C}>x_{-i C}$; otherwise Suspect $i$ will be charged more severely for crimes $\mathrm{B}$ and/or $\mathrm{C}$. The sentences for the two suspects are equitably calculated, therefore, the utility functions of the two suspects are symmetric.

In this game, every point in $[0, H-h] \times[0, T-t]$ is a possible strategy for each player (suspect). Then the set of strategies for each player is

$$
S_{i}=[0, H-h] \times[0, T-t], \quad \text { for } i=1,2 .
$$

For any given strategy profile $\left(\left(x_{i B}, x_{i C}\right),\left(x_{-i B}, x_{-i C}\right)\right) \in S_{i} \times S_{-i}$, Suspect $i$ will be fined an amount of (in dollars)

$$
h+2 t+\left(x_{i B}+2 x_{-i B}-\sqrt{x_{i B} x_{-i B}}\right)+2\left(x_{i C}+2 x_{-i C}-\sqrt{x_{i C} x_{-i C}}\right)
$$

and will be held in prison for (in days)

$$
\frac{h+\left(x_{i B}+2 x_{-i B}-\sqrt{x_{i B} x_{-i B}}\right)}{10 \sqrt{H}}+\frac{t+\left(x_{i C}+2 x_{-i C}-\sqrt{x_{i C} x_{-i C}}\right)}{\sqrt{T}} .
$$

More precisely, Suspect $i$ has the following utility function:

$$
\begin{aligned}
P_{i} & \left(\left(x_{i B}, x_{i C}\right),\left(x_{-i B}, x_{-i C}\right)\right) \\
= & \left(h+2 t+\left(x_{i B}+2 x_{-i B}-\sqrt{x_{i B} x_{-i B}}\right)+2\left(x_{i C}+2 x_{-i C}-\sqrt{x_{i C} x_{-i C}}\right),\right. \\
& \left.\frac{h+\left(x_{i B}+2 x_{-i B}-\sqrt{x_{i B} x_{-i B}}\right)}{10 \sqrt{H}}+\frac{t+\left(x_{i C}+2 x_{-i C}-\sqrt{x_{i C} x_{-i C}}\right)}{\sqrt{T}}\right)
\end{aligned}
$$

for $\left(\left(x_{i B}, x_{i C}\right),\left(x_{-i B}, x_{-i C}\right)\right) \in S_{i} \times S_{-i}$. By the given utility functions, the set of possible outcomes is

$$
U=[h+2 t, 2 H-h+2(T-t)] \times\left[\frac{h}{10 \sqrt{H}}+\frac{t}{\sqrt{T}}, \frac{2 H-h}{10 \sqrt{H}}+\frac{2 T-t}{\sqrt{T}}\right] .
$$

Suppose that, for each suspect, the amount of bribes accepted and the amount embezzled are not interchangeable. In addition, the amounts fined and the days sentenced to jail are not substitutable. Then the first part of the assumption implies that the sets of strategies 
$S_{i}$ are endowed with the coordinate ordering relation in 2-Euclidean space. That is, for $x_{i}=\left(x_{i 1}, x_{i 2}\right), y_{i}=\left(y_{i 1}, y_{i 2}\right) \in S_{i}$,

$$
x_{i} \succeq_{i} y_{i} \quad \text { if and only if } \quad x_{i 1} \geq y_{i 1} \text { and } x_{i 2} \geq y_{i 2} .
$$

Then $\left(S_{i}, \succeq_{i}\right)$ is a partially ordered topological space, for $i=1,2$.

From the second part of the assumption, we see that the suspects' preferences are reversed coordinate ordering relations on $R^{2}$. According to the suspects' preferences, an ordering relation $\succeq^{U}$ on $U$ is defined as: for $u=\left(u_{1}, u_{2}\right), v=\left(v_{1}, v_{2}\right) \in U$,

$$
u \succeq^{u} v \text { if and only if } \quad v_{1} \geq u_{1} \text { and } v_{2} \geq u_{2} .
$$

Then $\left(U, \succeq^{U}\right)$ is a poset (in fact, it is a partially ordered topological space with respect to the ordinal topology on $R^{2}$ ).

By optimizing the utility function, one can check that, for each Suspect $i$, the best response function $\beta_{i}: S_{-i} \rightarrow 2^{S_{i}} \backslash\{\varnothing\}$ is a single-valued function such that

$$
\beta_{i}\left(x_{-i B}, x_{-i C}\right)=\left(\frac{x_{-i B}}{4}, \frac{x_{-i C}}{4}\right), \quad \text { for all }\left(x_{-i B}, x_{-i C}\right) \in S_{-i} .
$$

Clearly, the two best response function $\beta_{1}$ and $\beta_{2}$ have a unique intersection point $((0,0),(0,0))$, which is the unique generalized Nash equilibrium of this game.

Example 5.3 (A different version of the two arrested venal officials' dilemma) Assume the same facts as Example 5.2 with the following utility functions instead:

$$
\begin{aligned}
P_{i} & \left(\left(x_{i B}, x_{i C}\right),\left(x_{-i B}, x_{-i C}\right)\right) \\
= & \left(h+2 t+\left(\frac{x_{i B}}{2}+2 x_{-i B}-\sqrt{x_{i B} x_{-i B}}\right)+2\left(\frac{x_{i C}}{2}+2 x_{-i C}-\sqrt{x_{i C} x_{-i C}}\right),\right. \\
& \left.\frac{h+\left(\frac{x_{i B}}{2}+2 x_{-i B}-\sqrt{x_{i B} x_{-i B}}\right)}{10 \sqrt{H}}+\frac{t+\left(\frac{x_{i C}}{2}+2 x_{-i C}-\sqrt{x_{i C} x_{-i C}}\right)}{\sqrt{T}}\right)
\end{aligned}
$$

for $\left(\left(x_{i B}, x_{i C}\right),\left(x_{-i B}, x_{-i C}\right)\right) \in S_{i} \times S_{-i}$. To find the best response function $\beta_{i}$ 's, simply consider a real valued function with variable $x, f(x)=\frac{x}{2}+2 y-\sqrt{x y}$, for some fixed nonnegative number $y$. Clearly,

$$
f(x)-f(y)=\frac{x}{2}+\frac{y}{2}-\sqrt{x y}=\frac{1}{2}(\sqrt{x}-\sqrt{y})^{2} \geq 0 .
$$

It implies that, for each Suspect $i$, the best response function $\beta_{i}: S_{-i} \rightarrow 2^{S_{i}} \backslash\{\varnothing\}$ is a singlevalued function

$$
\beta_{i}\left(x_{-i B}, x_{-i C}\right)=\left(x_{-i B}, x_{-i C}\right), \quad \text { for all }\left(x_{-i B}, x_{-i C}\right) \in S_{-i} .
$$

We find that, for any $\left(x_{1 B}, x_{2 C}\right) \in S_{1}$, the strategic profile $\left(\left(x_{1 B}, x_{2 C}\right),\left(x_{1 B}, x_{2 C}\right)\right)$ is a generalized Nash equilibrium of this game. Hence this game has infinitely many generalized Nash equilibria. 


\section{Competing interests}

The author declares that they have no competing interests.

\section{Acknowledgements}

The author is very grateful to the anonymous reviewers for their valuable suggestions, which improved the presentation of this paper.

Received: 14 May 2014 Accepted: 19 August 2014 Published: 5 September 2014

\section{References}

1. Li, JL: Several extensions of the Abian-Brown fixed point theorem and their applications to extended and generalized Nash equilibria on chain-complete posets. J. Math. Anal. Appl. 409, 1084-1092 (2014)

2. Carl, S, Heikkilä, S: Fixed Point Theory in Ordered Sets and Applications: From Differential and Integral Equations to Game Theory. Springer, New York (2010)

3. Li, JL: Extended Nash equilibria of nonmonetized strategic games on preordered sets. Int. J. Game Theory (to appear)

4. Xie, LS, Li, JL, Yang, WS: Order-clustered fixed point theorems on chain-complete preordered sets and their applications to extended and generalized Nash equilibria. Fixed Point Theory Appl. 2013(1/192), 1687-1812 (2013)

5. Xie, LS, Li, JL, Wen, CF: Applications of fixed point theory to extended Nash equilibriums of nonmonetized strategic games on posets. Fixed Point Theory Appl. 2013, 235 (2013). doi:10.1186/1687-1812-2013-235

6. Aliprantis, CD, Burkinshaw, O: Positive Operators. Springer, Dordrecht (2006)

7. Debreu, G: Representation of a preference ordering by a numerical function. In: Decision Processes. Cowles Foundation Paper, vol. 97. Wiley, New York (1954)

8. Dunford, N, Schwartz, JT: Linear Operators, Part I. Wiley, New York (1988)

9. Ok, EA: Order theory (forthcoming)

10. Ward, LE Jr.: Partially ordered topological space. Proc. Am. Math. Soc. 5(1), 144-161 (1954)

11. Takahashi, W: Nonlinear Functional Analysis. Yokohama Publisher, Yokohama (2000)

doi:10.1186/1687-1812-2014-192

Cite this article as: Li: Fixed point theorems on partially ordered topological vector spaces and their applications to equilibrium problems with incomplete preferences. Fixed Point Theory and Applications 2014 2014:192.

\section{Submit your manuscript to a SpringerOpen ${ }^{\circ}$ journal and benefit from:}

- Convenient online submission

- Rigorous peer review

- Immediate publication on acceptance

Open access: articles freely available online

- High visibility within the field

- Retaining the copyright to your article 